\title{
Vibrational entropy of spinodal decomposition in $\mathrm{FeCr}$
}

\author{
T. L. Swan-Wood, O. Delaire, and B. Fultz \\ California Institute of Technology, W. M. Keck Laboratory, Mail 138-78, Pasadena, California 91125, USA
}

(Received 2 February 2005; revised manuscript received 14 June 2005; published 25 July 2005)

\begin{abstract}
Inelastic neutron-scattering spectra were measured on stoichiometric $\mathrm{Fe}_{0.50} \mathrm{Cr}_{0.50}$ prepared as a bodycentered-cubic (bcc) solid solution, and after increasing amounts of chemical unmixing on the bcc lattice induced by annealing the solid solution at $773 \mathrm{~K}$. These spectra were reduced by a conventional procedure to a neutron-weighted vibrational density of states. Mössbauer spectrometry was used to characterize the extent of decomposition after annealing. A neutron-weight correction was performed, using results from the Mössbauer spectra and recent data on inelastic nuclear resonant scattering from ${ }^{57} \mathrm{Fe}-\mathrm{Cr}$. The vibrational entropy of decomposition was found to be $-0.17 \pm 0.01 k_{\mathrm{B}}$ /atom, nearly equal to the change in configurational entropy after spinodal decomposition. Effects of vibrational entropy on the thermodynamics of unmixing are analyzed, showing a large effect on the free energy with the formation of Cr-rich zones, and a large effect on the critical temperature for spinodal decomposition for equiatomic $\mathrm{Fe}_{0.50} \mathrm{Cr}_{0.50}$.
\end{abstract}

DOI: 10.1103/PhysRevB.72.024305 PACS number(s): 63.50.+x, 64.60.Cn, 81.30.Bx, 61.12. $-\mathrm{q}$

\section{INTRODUCTION}

Quantitative knowledge of energy, $E$, and entropy, $S$, is fundamental for the understanding of phase diagrams of materials, an active field of research that remains a grand challenge after decades of work. With this information, the Helmholtz free energy, $F=E-T S$, can be calculated, and the relative stabilities of different phases can be assessed at different temperatures, $T$. In the past decade, experimental and theoretical work has shown that vibrational entropy often makes a contribution to phase stability that is comparable to the classical configurational entropy. ${ }^{1-10}$ Vibrational entropy can significantly change the critical temperatures of phase transitions, but our understanding of systematic trends remains incomplete. The present work explores the vibrational entropy contribution to the chemical unmixing of $\mathrm{Fe}$ and $\mathrm{Cr}$ atoms on a bcc lattice that begins as a random solid solution of $\mathrm{Fe}_{0.50} \mathrm{Cr}_{0.50}$. The chemical energy prefers the unmixed state, while the configurational entropy of mixing prefers the mixed state. Although the conventional entropy of mixing is known to make an important contribution to the free energy, it was not clear if changes in vibrational entropy also make a significant contribution.

Unlike structural phase transitions that involve a transformation from one crystal structure to another, the chemical unmixing of Fe-Cr occurs on a bcc lattice with little change in lattice parameter. The process occurs by the continuous growth of composition modulations into Fe-rich and $\mathrm{Cr}$-rich regions, and is an example of "spinodal decomposition." Spinodal decomposition in alloys has been a phenomenon of much interest since the seminal presentation of Cahn and Hilliard of the nonlinear diffusion equation. ${ }^{11,12}$ Analytical research has addressed the nonlinearity of the equation, ${ }^{13-15}$ but today computers are capable of calculating the full diffusion equation. The kinetics of decomposition have been simulated, ${ }^{16-18}$ with some recent work based on the Onsager equation. ${ }^{19,20}$ Much experimental work on the $\mathrm{Fe}-\mathrm{Cr}$ system has addressed spinodal decomposition, owing to its correlation with the brittleness of ferritic stainless steels. ${ }^{21,22}$ The kinetics of decomposition has been of particular interest. ${ }^{23}$
Many experimental methods have been used for studying the composition modulations in $\mathrm{Fe}-\mathrm{Cr}$ alloys, including Mössbauer spectrometry ${ }^{24}$ and position-sensitive atom probe microscopy. ${ }^{25-28}$

Thermal neutron scattering from cubic crystals can be used to measure the phonon density of states (DOS), from which the vibrational entropy can be determined. Inelastic neutron scattering spectra from an alloy are generally distorted by differences in the scattering efficiencies of the elements. The measured spectra usually give a "neutronweighted DOS" (NWDOS). In the present work, an approach is used to correct for neutron weighting, giving a true DOS from which a vibrational entropy of decomposition is assessed as $-0.17 \pm 0.01 k_{\mathrm{B}}$ /atom. This vibrational entropy is approximately equal to the configurational entropy of decomposition, and makes an important contribution to the thermodynamics of forming $\mathrm{Cr}$-rich zones. By including the vibrational entropy in the free energy, the critical temperature for spinodal decomposition is lowered by $20 \%$ of its value predicted with configurational entropy alone.

\section{EXPERIMENTAL}

Alloys of stoichiometric $\mathrm{Fe}_{0.50} \mathrm{Cr}_{0.50}$ were prepared by arc-melting under an argon atmosphere, using shots of 99.99\% Fe and $99.995 \%$ Cr. There was negligible mass loss and little visible surface oxidation after melting, so the compositions are expected to be accurate to 0.1 at. $\%$. The ingots were cold rolled to a thickness of $1.5 \mathrm{~mm}$, as appropriate for scattering $10 \%$ of the incident neutrons. The rolled strips were heated to $1273 \mathrm{~K}$, and quenched into iced brine to prepare disordered solid solutions. Some samples were then heat treated to obtain various states of chemical unmixing by annealing at $773 \mathrm{~K}$ in argon for 12,46 , and $433 \mathrm{~h}$, followed by air cooling.

$\mathrm{X}$-ray diffraction patterns were acquired from all samples using an Inel CPS-120 diffractometer with $\mathrm{Co} \mathrm{K} \alpha$ radiation and a position-sensitive detector. These data confirmed that the crystal structure was body centered cubic (bcc), with no 
evidence of the sigma phase after annealing at $773 \mathrm{~K}$.

Mössbauer spectrometry was performed on all materials at room temperature using a conventional constant acceleration spectrometer with a $10 \mathrm{mCi}$ source of ${ }^{57} \mathrm{Co}$ in $\mathrm{Rh}$. The effects of first- and second-nearest-neighbor $\mathrm{Cr}$ atoms on the

${ }^{57} \mathrm{Fe}$ hyperfine magnetic field have been used for many years to determine the extent of spinodal decomposition in $\mathrm{Fe}-\mathrm{Cr}$ alloys, and our results were consistent with previous experimental data. ${ }^{24} \mathrm{~A}$ semiquantitative interpretation of these spectra is presented in Sec. III A.

Time-of-flight inelastic neutron scattering data were acquired with both the low resolution medium energy chopper spectrometer (LRMECS) and high resolution medium energy chopper spectrometer (HRMECS) instruments at the Intense Pulsed Neutron Source at the Argonne National Laboratory. Samples for LRMECS and HRMECS were mounted in thinwall aluminum cans, $7 \mathrm{~cm} \times 10 \mathrm{~cm}$ and $10 \mathrm{~cm} \times 11 \mathrm{~cm}$, respectively, using incident beams of monochromatic neutrons with energies of $55 \mathrm{meV}$ and $60 \mathrm{meV}$, respectively. The flat sample packages were tilted at a $45^{\circ}$ angle off the direction of the incident beam to minimize self-shielding. All spectra were measured at room temperature. Background data were acquired from the empty aluminum cans under the same conditions.

The raw data of intensity versus time of flight in the different detector tubes were reprocessed into intensity versus energy, and then converted into an approximate or "neutronweighted" phonon density-of-states (NWDOS) using the following steps. The raw data were corrected for detector efficiency using a white beam spectrum from a vanadium plate, and normalized by the incident flux on the samples. The time-independent background was removed. The timedependent parts of the background acquired from the empty cans were then subtracted. Detector data were rebinned into angular bins of $10^{\circ}$ each, using scattering angles up to $120^{\circ}$ for LRMECS data, and $133^{\circ}$ for HRMECS data. The lowangle data (angles less than approximately $40^{\circ}$ ) were neglected to minimize contributions from magnetic scattering. After subtraction of an elastic peak centered about $0 \mathrm{meV}$, the data below approximately $5 \mathrm{meV}$ were approximated as a straight line, as expected in the continuum limit. Corrections were then made for thermal factors and multiphonon scattering (two- through five-phonon processes), using an iterative procedure in the incoherent approximation. ${ }^{29}$ The result is a phonon density of states (DOS), but an approximate one that we call $g^{\mathrm{nw}}(E)$ because the cross sections for phonon scattering by $\mathrm{Fe}$ and $\mathrm{Cr}$ atoms are not equal. Our correction for this "neutron weighting" is described below.

\section{RESULTS}

\section{A. Mössbauer spectrometry}

The Mössbauer spectrum of bcc pure Fe is one sextet of absorption peaks, caused by the nuclear Zeeman splitting of the nuclear spin levels in the hyperfine magnetic field (HMF) at the nucleus. Mössbauer spectra of Fe-rich ferromagnetic bcc alloys show clear perturbations of ${ }^{57} \mathrm{Fe} \mathrm{HMFs}$ caused by neighboring $\mathrm{Cr}$ atoms, and ${ }^{57} \mathrm{Fe}$ atoms with different numbers of $\mathrm{Cr}$ neighbors contribute subspectra of sextets having different separations in Doppler velocity. Those ${ }^{57} \mathrm{Fe}$ atoms with $\mathrm{Cr}$ atoms in their first- and second-nearest-neighbor (1NN and $2 \mathrm{NN}$ ) shells have a reduction in HMF of approximately $27 \mathrm{kG}$ for each $\mathrm{Cr}$ neighbor. ${ }^{30}$ The integer $m$, where $0 \leqslant m \leqslant 14$, denotes the number of $1 \mathrm{NN}$ and $2 \mathrm{NN} \mathrm{Cr}$ neighbors about a specific ${ }^{57} \mathrm{Fe}$ atom. The HMF, $H(m)$, can be calculated reasonably well by adding these HMF perturbations from neighboring $\mathrm{Cr}$ atoms, ${ }^{30,33,34}$

$$
H(m, \chi)=H_{0}+m \Delta H_{1,2}^{\mathrm{Cr}}+\kappa \chi,
$$

where $H_{0}$ is the $\mathrm{HMF}$ of ${ }^{57} \mathrm{Fe}$ in pure bcc Fe, and each $1 \mathrm{NN}$ or $2 \mathrm{NN} \mathrm{Cr}$ atom perturbs the HMF by the amount $\Delta H_{1,2}^{\mathrm{Cr}}$. The additional contribution $\kappa \chi$, where $\chi$ is the concentration of $\mathrm{Cr}$ and $\kappa$ is a constant, accounts approximately for the effects of $\mathrm{Cr}$ atoms beyond the $1 \mathrm{NN}$ and $2 \mathrm{NN}$ shells, but these effects are relatively small. Neighboring $\mathrm{Cr}$ atoms also cause an isomer shift by the amount of $\Delta i=-0.02 \mathrm{~mm} / \mathrm{sec}$ per $\mathrm{Cr}$ neighbor, shifting slightly all peaks in a sextet to lower velocities than the pure Fe spectrum by the amount $m \Delta i$. For a fixed alloy concentration, the positions of all subspectral sextets are fully specified by the number of $\mathrm{Cr}$ neighbors $m$, and the characteristics of the nuclear Zeeman effect. Owing to a tendency for the sample magnetization to lie within the plane of the flat sample, the intensities of the six peaks in the sextet were found to be approximately 3.0:3.0:1.0:1.0:3.0:3.0.

Calculating a complete Mössbauer spectrum also requires information on the fractions of ${ }^{57} \mathrm{Fe}$ atoms having different numbers of $\mathrm{Cr}$ neighbors, $m$. For a bcc random solid solution of $\mathrm{Cr}$ concentration $\chi$, the probability that a ${ }^{57} \mathrm{Fe}$ atom will have $m$ solute neighbors in any of the 14 sites of its $1 \mathrm{NN}$ and $2 \mathrm{NN}$ shells is the binomial probability, $P(14, m, \chi)$,

$$
P(14, m, \chi)=\frac{14 !}{m !(14-m) !} \chi^{m}(1-\chi)^{14-m} .
$$

Using Eq. (1) and its analog for isomer shifts, and Eq. (2) with trial values of $\chi$, sextets were calculated. These were fit to the measured Mössbauer spectra to obtain optimal values of $\chi$, where $\chi$ is the $\mathrm{Cr}$ concentration in the Fe-rich region. This procedure is most easily justified for the quenched sample, which we believe to be a disordered solid solution. Unfortunately, the additive perturbation model becomes unreliable for Cr-rich regions, especially for regions that lose their ferromagnetism. Fortunately, these regions do not contribute to the outer parts of the Mössbauer spectrum (around peaks 1 and 6 at higher Doppler velocities), which become more distinct with further annealing. The fits for the samples that were annealed for $12 \mathrm{~h}$ and $46 \mathrm{~h}$ included spectra for both the Fe-rich and Cr-rich regions. For the spectrum from the sample annealed for $433 \mathrm{~h}$, the interpretation is simpler, because it appears that the $\mathrm{Cr}$-rich regions contribute primarily a paramagnetic peak in the center of the spectrum, with a peak intensity consistent with an Fe concentration of 20 at. \%. Our measured spectrum is somewhat broader than a spectrum measured previously on $\mathrm{Fe}_{0.40} \mathrm{Cr}_{0.60}$ annealed for 433 hours at $773 \mathrm{~K},{ }^{24,31}$ consistent with the differences in composition. The experimental Mössbauer spectra from all samples are presented in Fig. 1, together with the calculated best fits shown as dashed lines and labeled with the optimum 


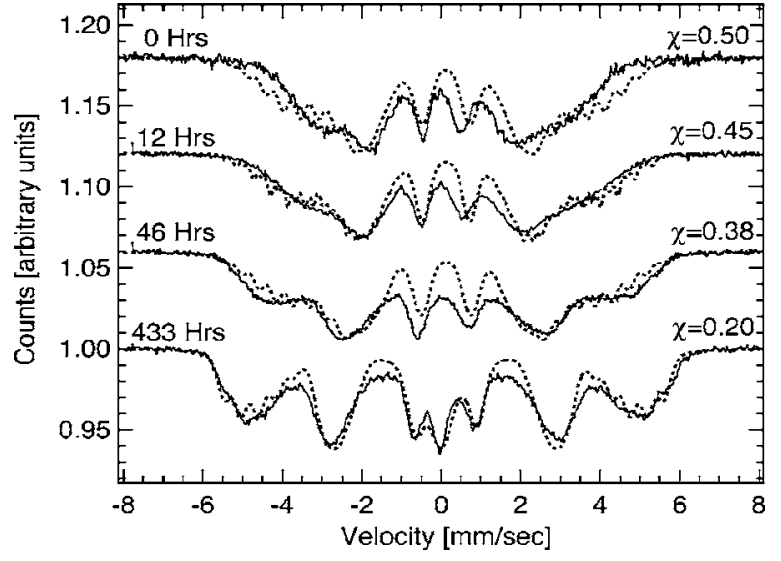

FIG. 1. Experimental Mössbauer data from all samples (solid lines). The additive perturbation model was used to fit the experimental spectra (dotted lines). These fits to spectra are labeled by the $\mathrm{Cr}$ concentrations, $\chi$, of the Fe-rich regions.

value of $\chi$ used for the calculation. The measured $\chi$ is an average composition for the Fe-rich regions, without details on the modulations of the chemical composition in the partially unmixed samples.

\section{B. Inelastic neutron scattering}

Each atomic species, $d$, of concentration $\chi_{d}$ in an alloy has a partial density of states (PDOS), $g_{d}^{\chi}$, which contributes to the total DOS, $g(E)$,

$$
g(E)=\sum_{d} g_{d}^{\chi}(E) \chi_{d}
$$

"Neutron weighting" distorts experimental spectra because phonon scattering by different isotopes occurs with different efficiencies. What is measured by inelastic neutron scattering is the "neutron-weighted" DOS (NWDOS), which is a weighted linear combination of the PDOS,

$$
g^{\mathrm{nw}}(E) \propto \exp (2 W) \sum_{d} g_{d}^{\chi}(E) \exp \left(-2 W_{d}\right) \frac{\sigma_{d}}{M_{d}},
$$

where $\exp \left(-2 W_{d}\right)$ is the Debye-Waller factor, $\sigma_{d}$ is the total scattering cross section, and $M_{d}$ is the mass of atom $d$. The factor $\exp (2 W)$ is the average Debye-Waller correction calculated from the self-consistent NWDOS. We assume $\exp \left[-2\left(W-W_{d}\right)\right] \simeq 1$, an assumption that is most reliable at low temperatures, so

$$
g^{\mathrm{nw}}(E)=A \sum_{d} g_{d}^{\chi}(E) \frac{\sigma_{d}}{M_{d}} x_{d},
$$

where the normalization factor, $A$, depends on the concentrations of the elements and their scattering strengths,

$$
A=\left(\sum_{d} \frac{\sigma_{d}}{M_{d}} \chi_{d}\right)^{-1} .
$$

If the neutron-weighting factor, $\sigma_{d} / M_{d}$, in Eq. (5) were equal for both $\mathrm{Fe}$ and $\mathrm{Cr}$, then Eq. (5) would equal Eq. (3). In our case, phonon scattering from natural $\mathrm{Fe}$ is three times stronger than from natural $\mathrm{Cr}$, or $\sigma_{\mathrm{Fe}} / M_{\mathrm{Fe}}=3 \sigma_{\mathrm{Cr}} / M_{\mathrm{Cr}}$. The iterative technique, described in Sec. II, produces a NWDOS, where the Fe PDOS is overweighted by a factor of 3. Phonon modes with large displacements of $\mathrm{Fe}$ atoms would make larger contributions to the measured spectra than would modes where $\mathrm{Cr}$ motions were dominant. This would distort the NWDOS from the true DOS if the Fe PDOS differs significantly from the Cr PDOS.

\section{Neutron-weight correction}

Keune and Sturhahn ${ }^{35}$ and Ruckert et al. ${ }^{36}$ performed inelastic nuclear resonant x-ray scattering (INRXS) on $\mathrm{Fe} / \mathrm{Cr}$ multilayer thin films prepared by molecular beam epitaxy on (100) bcc surfaces. INRXS measures scattering only from ${ }^{57} \mathrm{Fe}$, and can provide the ${ }^{57} \mathrm{Fe}$ PDOS. These measurements produced the PDOS for ${ }^{57} \mathrm{Fe}$ monolayers separated from $\mathrm{Cr}$ layers by inactive layers of ${ }^{56} \mathrm{Fe}$. Table I lists the layering order for each of their INRXS samples.

Equation (3) showed that the sum of the PDOS from Fe and $\mathrm{Cr}$ is the total DOS needed for thermodynamic calculations. Although PDOSs from $\mathrm{Fe}$ or $\mathrm{Cr}$ are not available for disordered and partially unmixed $\mathrm{FeCr}$, we can utilize the INRXS results to construct an Fe PDOS if we know the effect of $\mathrm{Cr}$ neighbors on the $\mathrm{Fe}$ PDOS. The $\mathrm{Fe}$ and $\mathrm{Cr}$ interatomic force constants are much larger for first and second neighbors than more distant neighbors in the bcc structure, ${ }^{37,38}$ so we parametrize the Fe PDOS by the same average number of $\mathrm{Cr}$ atoms in the $1 \mathrm{NN}$ and $2 \mathrm{NN}$ shells, $m$, that is obtained by Mössbauer spectrometry. We therefore approximate the Fe PDOS as a sum of contributions from $\mathrm{Fe}$ atoms with different numbers of $\mathrm{Cr}$ neighbors

$$
g_{\mathrm{Fe}}^{\chi}(E)=\sum_{m \in[0,1,2, . .14]} P(14, m, \chi) g_{\mathrm{Fe}}^{m}(E),
$$

where $g_{\mathrm{Fe}}^{m}(E)$ is the Fe PDOS for an Fe atom with $m \mathrm{Cr}$ neighbors in its $1 \mathrm{NN}$ and $2 \mathrm{NN}$ shells, and $P(14, m, \chi)$ and $\chi$ are the same binomial probability and $\mathrm{Cr}$ concentration used in Eq. (2). Because the INRXS data are available for only four samples, each with an average of $\mu \mathrm{Cr}$ atoms in the ${ }^{57} \mathrm{Fe}$ $1 \mathrm{NN}$ and $2 \mathrm{NN}$ shells, we assign the same measured INRXS spectrum to a set of individual $m$, referring to these sets as $\{m\}_{\mu}$. The average of each set, $\{m\}_{\mu}$, is the value $\mu$. The probabilities are conserved,

$$
\sum_{m} P(14, m, \chi)=\sum_{\mu}\left[\sum_{m \in\{m\}_{\mu}} P(14, m, \chi)\right]=1 .
$$

Table I lists the groupings of $m$ into $\{m\}_{\mu}$. For the compositions relevant to the present study, the table gives the probabilities of the sets, $\{m\}_{\mu}$, i.e., the sums $\Sigma_{m \in\{m\}_{\mu}} P(14, m, \chi)$. With this coarse-graining procedure, we express the Fe PDOS of a disordered alloy in terms of the PDOSs from the INRXS spectra,

$$
g_{\mathrm{Fe}}^{\chi}(E)=\sum_{\mu}\left[\sum_{m \in\{m\}_{\mu}} P(M=14, m, \chi)\right] g_{\mathrm{Fe}}^{\mu}(E),
$$

where $g_{\mathrm{Fe}}^{\mu}(E)$ is the normalized Fe PDOS from the INRXS samples. 
TABLE I. Properties of Fe-Cr multilayer samples of prior inelastic nuclear-resonant $\mathrm{x}$-ray scattering measurements (Ref. 36). In samples $\mathrm{FeCr} 1, \mathrm{FeCr} 2$, and $\mathrm{FeCr} 3$ the ${ }^{57} \mathrm{Fe}$ layer is a monolayer thick. $\mathrm{FeCr} 4$ is a bulk alloy of ${ }^{57} \mathrm{Fe}_{0.03} \mathrm{Cr}_{0.97}$.

\begin{tabular}{|c|c|c|c|c|}
\hline Label & $\mathrm{FeCr} 1$ & $\mathrm{FeCr} 2$ & $\mathrm{FeCr} 3$ & $\mathrm{FeCr} 4$ \\
\hline Layering order & ${ }^{56} \mathrm{Fe} /{ }^{57} \mathrm{Fe} /{ }^{56} \mathrm{Fe}$ & $\mathrm{Cr} /{ }^{57} \mathrm{Fe} /{ }^{56} \mathrm{Fe}$ & $\mathrm{Cr} /{ }^{57} \mathrm{Fe} / \mathrm{Cr}$ & ${ }^{57} \mathrm{Fe}_{0.03} \mathrm{Cr}_{0.97}$ \\
\hline$\mu$ & 0 & 5 & 10 & 13.58 \\
\hline$\{m\}_{\mu}$ & {$[0,1,2]$} & {$[3,4,5,6,7,8]$} & {$[9,10,11]$} & {$[12,13,14]$} \\
\hline$\sum_{m \in\{m\}_{\mu}} P(\chi=0.20)$ & 0.448 & 0.552 & 0.000 & 0.000 \\
\hline$\sum_{m \in\{m\}_{\mu}} P(\chi=0.38)$ & 0.054 & 0.904 & 0.042 & 0.000 \\
\hline$\sum_{m \in\{m\}_{\mu}} P(\chi=0.45)$ & 0.017 & 0.864 & 0.117 & 0.002 \\
\hline$\sum_{m \in\{m\}_{\mu}} P(\chi=0.50)$ & 0.006 & 0.782 & 0.206 & 0.006 \\
\hline$\sum_{m \in\{m\}_{\mu}} P(\chi=0.55)$ & 0.002 & 0.661 & 0.320 & 0.017 \\
\hline$\sum_{m \in\{m\}_{\mu}} P(\chi=0.62)$ & 0.000 & 0.452 & 0.494 & 0.054 \\
\hline$\sum_{m \in\{m\}_{\mu}} P(\chi=0.80)$ & 0.000 & 0.044 & 0.508 & 0.448 \\
\hline
\end{tabular}

Equation (9) is an approximation for the Fe PDOS of a random bcc $\mathrm{Fe}-\mathrm{Cr}$ binary alloy with $\mathrm{Cr}$ concentration $\chi$, and can be used in a neutron-weight correction for the disordered solid solution for which $\chi=0.5$ [using Eqs. (3) or (5). For the unmixed materials, however, it is necessary to account for the presence of $\mathrm{Fe}$-rich and $\mathrm{Cr}$-rich regions. For example, for the sample annealed for $433 \mathrm{~h}$, we use a $50 \%$ volume fraction of $\chi=0.2$ and a $50 \%$ volume fraction of $\chi=0.8$, approximately consistent with the intensity of the paramagnetic peak in the Mössbauer spectrum. For this material the Fe PDOS was weighted by the fractions of $\mathrm{Fe}$ in the two regions

$$
g_{\mathrm{Fe}}^{\chi}(E) \chi_{\mathrm{Fe}}=\sum_{\chi=0.2,0.8} \chi \nu_{\chi} g_{\mathrm{Fe}}^{\chi}(E)
$$

where $\nu_{\chi}$, approximately $1 / 2$, is the mole fraction associated with each $\chi$. Equation (10) is in a form suitable for a neutron weight correction with Eqs. (3) or (5). Using the INRXS spectra from Keune and Sturhahn ${ }^{35}$ and Ruckert et al. ${ }^{36}$ we find as expected that with increased unmixing, the shape of $g_{\mathrm{Fe}}^{\chi}(E)$ approaches closely the DOS of pure bcc Fe. This Fe PDOS was used along with the known weighting factors, $\chi \sigma / M$, to isolate the Cr PDOS from the measured data. The Cr PDOS was found to have somewhat higher phonon frequencies than the Fe PDOS. Once the Cr PDOS was isolated, the true, unweighted DOS, $g(E)$, was calculated with Eq. (3).
These corrected DOS curves are presented in Fig. 2 and Fig. 3.

The DOS curves undergo an overall stiffening that increases with the extent of chemical unmixing, shifting the cutoff energy by approximately $2.0 \mathrm{meV}$ for the 433-hour sample. This stiffening gives a reduction in the vibrational entropy. The harmonic vibrational entropy is calculated as

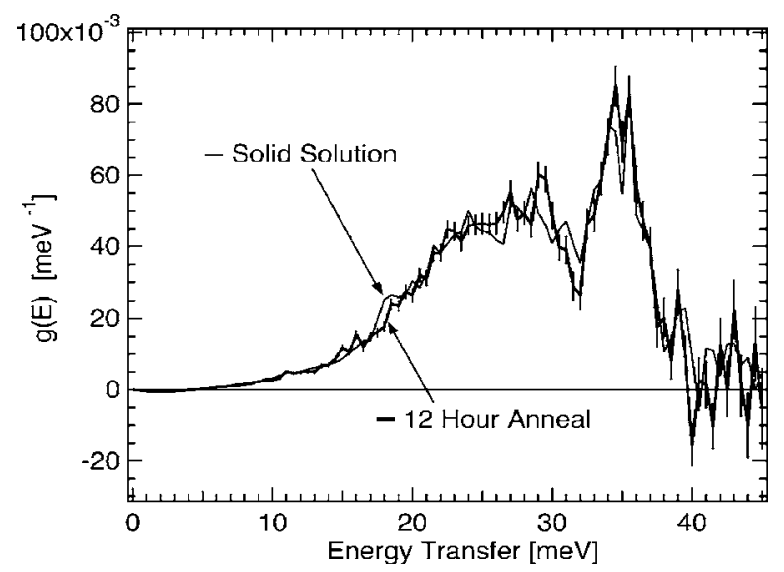

FIG. 2. Neutron-weight-corrected density of states of FeCr from spectra measured on LRMECS at the Intense Pulsed Neutron Source. 


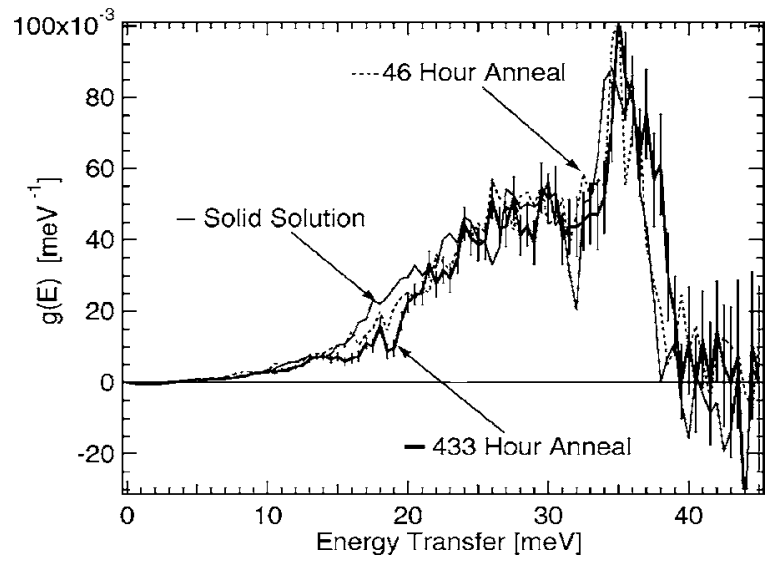

FIG. 3. Neutron-weight-corrected density of states of $\mathrm{FeCr}$ from spectra measured on HRMECS at the Intense Pulsed Neutron Source.

$$
S_{\mathrm{vib}}(T)=3 k_{\mathrm{B}} \int_{0}^{\infty} g(E)\left[\left(n_{E}+1\right) \ln \left(n_{E}+1\right)-n_{E} \ln \left(n_{E}\right)\right] d E,
$$

where and $n_{E}(T)$ is the Bose-Einstein Factor. The vibrational entropy of spinodal decomposition was calculated as the difference in $S_{\text {vib }}(773 \mathrm{~K})$ between the annealed sample and the sample prepared as a solid solution. These differences were $-0.01 \pm 0.01 k_{\mathrm{B}} /$ atom, $\quad-0.035 \pm 0.01 k_{\mathrm{B}} /$ atom, and $-0.17 \pm 0.01 k_{\mathrm{B}} /$ atom for the samples annealed for 12,46 , and $433 \mathrm{~h}$, respectively. The error bars represent the statistical uncertainty in the measured data. The error from the neutronweight correction cannot be calculated quantitatively. The neutron-weight correction increased the magnitude of the vibrational entropy of unmixing by a factor of approximately 1.8 .

\section{DISCUSSION}

\section{A. Thermodynamics of unmixing}

The configurational entropy of a fully disordered binary alloy of solute concentration $\chi$ is

$$
S_{\mathrm{cf}}^{\mathrm{pt}}=-k_{\mathrm{B}}[(1-\chi) \ln (1-\chi)+\chi \ln \chi] .
$$

For real alloys, the point (or Bragg-Williams) approximation of Eq. (12) generally overestimates the configurational entropy because it neglects short-range correlations that constrain the configurations available to the system. A previous x-ray diffuse-scattering study on $\mathrm{Fe}_{0.53} \mathrm{Cr}_{0.47}$ found some clustering in the alloy. ${ }^{32}$ To use Eq. (12) to calculate the configurational entropy for an unmixed alloy, we assume two well-separated regions with the distinct compositions of Table I. In reality, however, there will be a spread of compositions of the Fe-rich and Cr-rich zones. Because a Mössbauer spectrum gives an average $\mathrm{Cr}$ concentration for the Fe-rich zones, the averaging overcomposition is already included to some extent. For the solid solution sample of $\mathrm{Fe}_{0.50} \mathrm{Cr}_{0.50}$ and for the most unmixed sample of $\mathrm{Fe}_{0.80} \mathrm{Cr}_{0.20}$ plus $\mathrm{Fe}_{0.20} \mathrm{Cr}_{0.80}$, Eq. (12) gives a maximum possible change

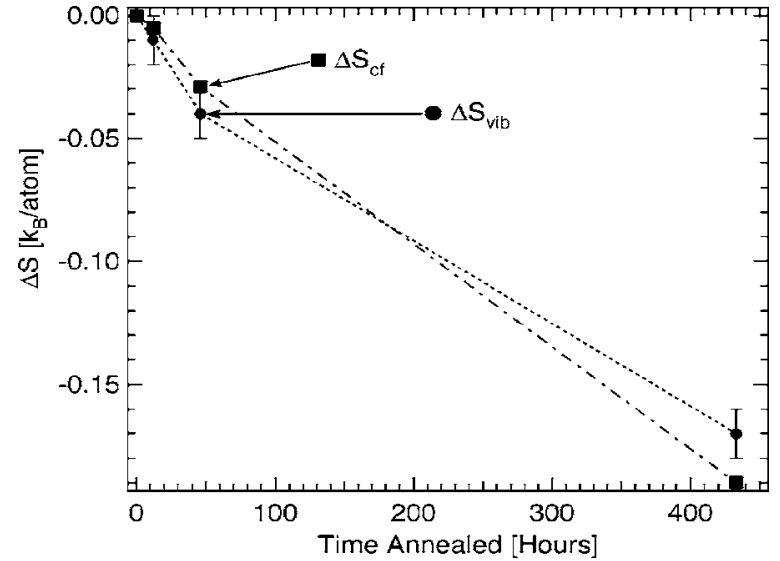

FIG. 4. Vibrational and configurational entropy of unmixing as a function of time annealed at $773 \mathrm{~K}$.

in configurational entropy, $\Delta S_{\mathrm{cf}}^{\mathrm{pt}}=-0.19 k_{\mathrm{B}} /$ atom. Thus, $\Delta S_{\text {vib }} \approx \Delta S_{\text {cf }}$, and vibrational entropy is a significant part of the entropy change during spinodal decomposition. Figure 4 compares the vibrational and configurational entropy of decomposition as a function of decomposition.

The enthalpy of mixing of $\mathrm{Fe}-\mathrm{Cr}$ has been measured by high-temperature calorimetry, ${ }^{39}$ and was assessed with recent first-principles calculations. ${ }^{40}$ Unmixing from a solid solution of $\mathrm{Fe}_{0.50} \mathrm{Cr}_{0.50}$ to equal mole fractions of $\mathrm{Fe}_{0.20} \mathrm{Cr}_{0.80}$ and $\mathrm{Fe}_{0.80} \mathrm{Cr}_{0.20}$ causes a decrease of enthalpy, $\Delta E=-2 \mathrm{~kJ} / \mathrm{mole}$. For comparison, the product $T \Delta S_{\mathrm{vib}}=-1.1 \mathrm{~kJ} / \mathrm{mole}$ at $T$ $=773 \mathrm{~K}$. Vibrational entropy makes an important contribution to the free energy of unmixing.

\section{B. Critical temperature of spinodal decomposition}

Spinodal decomposition in a binary alloy of solute concentration $\chi$ occurs below a critical temperature $T_{\mathrm{C}}$ at which the curvature of the free energy with respect to concentration is zero at $\chi$. The fractional change of the critical temperature by including the vibrational entropy with the configurational entropy, as opposed to the configurational entropy alone, is

$$
\frac{T_{\mathrm{Ccf}}-T_{\mathrm{C}}}{T_{\mathrm{Ccf}}}=\frac{S_{\mathrm{vib}}^{\prime \prime}(\chi)}{S_{\mathrm{cf}}^{\prime \prime}(\chi)+S_{\mathrm{vib}}^{\prime \prime}(\chi)},
$$

where $T_{\mathrm{Ccf}}$ is the critical temperature assuming all entropy is configurational, $T_{\mathrm{C}}$ is the critical temperature assuming entropy is configurational and vibrational, $S_{\mathrm{vib}}^{\prime \prime}(\chi)$ is the second derivative of the vibrational entropy with respect to $\chi$, and $S_{\mathrm{cf}}^{\prime \prime}(\chi)$ is the second derivative of the configurational entropy with respect to $\chi$.

To assess the effect of vibrational entropy on the critical temperature for unmixing through Eq. (13), we used Eq. (12) to obtain the curvature $S_{\mathrm{cf}}^{\prime \prime}(\chi=0.5)=-4 k_{\mathrm{B}} /$ atom. Measurements of $S_{\text {vib }}$ at different $\mathrm{Cr}$ concentrations are needed to obtain the vibrational entropy as a function of $\chi$, and then its curvature. $\mathrm{Fe}$ and $\mathrm{Cr}$ force constants were obtained by fitting triple-axis measured phonon dispersions to a set of $\mathrm{Fe}-\mathrm{Cr}$ alloys, and these force constants were used in a Born von Kármán simulation to calculate a DOS. ${ }^{41}$ These DOS curves gave vibrational entropies shown in Fig. 5, from which 


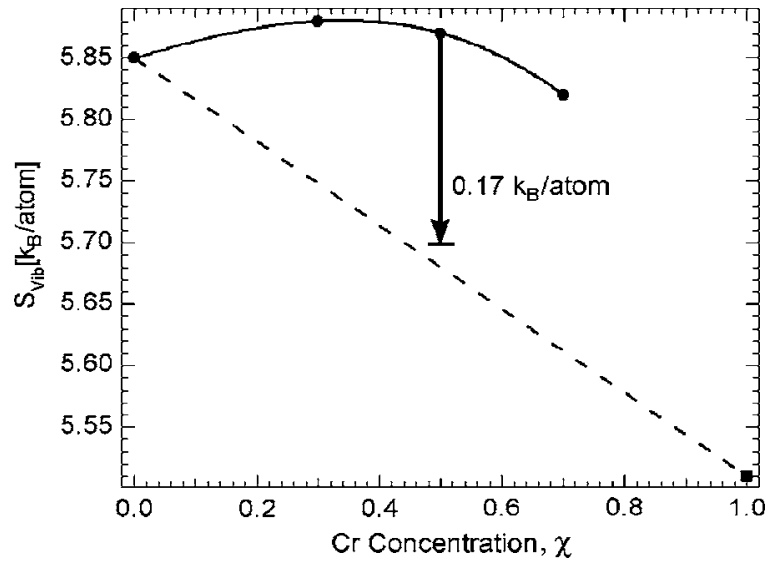

FIG. 5. Vibrational entropy versus $\mathrm{Cr}$ concentration, $S_{\text {vib }}(\chi)$, calculated in the harmonic approximation. The five data points are from triple-axis measurements on $\mathrm{Fe}$ and $\mathrm{Fe}_{1-x} \mathrm{Cr}_{x}$ alloys (Ref. 41) and $\mathrm{Cr}$ (Ref. 38). The dashed line connects elemental $\mathrm{Fe}$ and $\mathrm{Cr}$. The third-order polynomial fit to the alloy data is shown as the solid line and was used to obtain $S_{\mathrm{vib}}^{\prime \prime}(\chi=0.5)$. The magnitude of the measured vibrational entropy of unmixing, $-0.17 k_{\mathrm{B}} /$ atom, is shown and labeled.

we obtain an approximate numerical value $S_{\text {vib }}^{\prime \prime}(\chi=0.5)$ $=-1.0 k_{\mathrm{B}} /$ atom. With these curvatures, Eq. (13) predicts a decrease in critical temperature for spinodal decomposition of $20 \%$. This is a large effect, and is consistent with the large change in vibrational entropy during spinodal decomposition that was measured in the present work.

The low vibrational entropy of the Cr-rich regions does not occur until high $\mathrm{Cr}$ concentrations are reached, perhaps correlating with the loss of ferromagnetism. This is consistent with the larger stiffening of phonon DOS for the alloy heat treated for $433 \mathrm{~h}$, which showed a paramagnetic peak in its Mössbauer spectrum. The change in vibrational entropy after 433 hours $\left(-0.17 k_{\mathrm{B}} /\right.$ atom $)$, is almost precisely the difference between the entropy of the solid solution and the average entropy of pure $\mathrm{Fe}$ and pure $\mathrm{Cr}$, as shown by Fig. 5 .

The present results, taken with the previous results, ${ }^{41}$ indicate that the vibrational entropy does not change significantly until zones are formed with high $\mathrm{Cr}$ concentration, causing these zones to be destabilized somewhat owing to a higher free energy. The phase boundary for spinodal decomposition therefore should be asymmetrical about the composition of $\chi=0.5$, being suppressed to lower temperatures at high $\mathrm{Cr}$ concentrations. It is possible that the kinetics may be slowed in the later stages of spinodal decomposition owing to this asymmetry of vibrational entropy, but many other factors affect kinetic processes besides the change in free energy. Further study of the vibrational entropy in alloys of high $\mathrm{Cr}$ concentration would be appropriate.

\section{CONCLUSIONS}

Inelastic neutron-scattering spectra were measured on stoichiometric FeCr samples with increasing degrees of chemical unmixing, and analyzed to give phonon density of states curves. It was found that all vibrational modes of the density of states stiffen monotonically as decomposition progresses. Mössbauer spectra confirmed the spinodal decomposition of the samples, and gave the average $\mathrm{Cr}$ concentration from the Fe-rich regions through an analysis of hyperfine magnetic fields. A neutron-weight correction was performed, giving the vibrational entropy of decomposition to be $-0.17 \pm 0.01 k_{\mathrm{B}}$ /atom after $433 \mathrm{~h}$ of annealing at $773 \mathrm{~K}$. In the point approximation, the configurational entropy of decomposition is $-0.19 k_{\mathrm{B}} /$ atom, so the vibrational entropy of decomposition is approximately equal to the configurational entropy of decomposition. The vibrational entropy changes greatly when zones with high $\mathrm{Cr}$ concentration are formed, causing these zones to be destabilized somewhat owing to an overall stiffening of phonon modes. Near the equiatomic composition of $\mathrm{Fe}_{0.50} \mathrm{Cr}_{0.50}$, the vibrational entropy has a significant dependence on concentration, causing the critical temperature of spinodal decomposition of $\mathrm{Fe}_{0.50} \mathrm{Cr}_{0.50}$ to decrease by $20 \%$.

\section{ACKNOWLEDGMENTS}

This work was supported by the U.S. Department of Energy through the Basic Energy Sciences Grant Nos.DEFG02-03ER46055 and BES-MS, W-31-109-ENG-38.
${ }^{1}$ L. Anthony, J. K. Okamoto, and B. Fultz, Phys. Rev. Lett. 70, 1128 (1993).

${ }^{2}$ G. D. Garbulsky and G. Ceder, Phys. Rev. B 49, 6327 (1994).

${ }^{3}$ B. Fultz, L. Anthony, L. J. Nagel, R. M. Nicklow, and S. Spooner, Phys. Rev. B 52, 3315 (1995).

${ }^{4}$ E. Obradó, L. Mañosa, and A. Planes, Phys. Rev. B 56, 20 (1997).

${ }^{5}$ V. Ozolinšs, C. Wolverton, and A. Zunger, Phys. Rev. B 58, R5897 (1998).

${ }^{6}$ P. D. Bogdanoff and B. Fultz, Philos. Mag. B 79, 753 (1999).

${ }^{7}$ P. D. Bogdanoff and B. Fultz, Philos. Mag. B 81, 299 (2001).

${ }^{8}$ V. Ozoliņš and M. Asta, Phys. Rev. Lett. 86, 448 (2001).

${ }^{9}$ C. Wolverton and V. Ozoliņš, Phys. Rev. Lett. 86, 5518 (2001).
${ }^{10}$ A. van de Walle and G. Ceder, Rev. Mod. Phys. 74, 11 (2002).

${ }^{11}$ J. W. Cahn and J. E. Hilliard, J. Chem. Phys. 28, 258 (1958).

${ }^{12}$ J. W. Cahn and J. E. Hilliard, J. Chem. Phys. 31, 688 (1959).

${ }^{13}$ L. A. Swanger, P. K. Gupta, and A. R. Cooper, Jr., Acta Metall. 18, 9 (1970).

${ }^{14}$ J. S. Langer, M. Baron, and H. D. Miller, Phys. Rev. A 11, 1417 (1975).

${ }^{15}$ T. Tsakalakos, Scr. Metall. 15, 255 (1981).

${ }^{16}$ H. Nishimori and A. Onuki, Phys. Rev. B 42, 980 (1990).

${ }^{17}$ T. Miyazaki, A. Takeuchi, and T. Koyama, J. Mater. Sci. 27, 2444 (1992).

${ }^{18}$ A. Takeuchi, T. Koyama, and T. Miyazaki, J. Jpn. Inst. Met. 57, 492 (1993). 
${ }^{19}$ Y. Wang, L. Q. Chen, and A. G. Khachaturyan, Acta Metall. Mater. 41, 279 (1993).

${ }^{20}$ T. Koyama, T. Miyazaki, and M. Mebed, Metall. Mater. Trans. A 26A, 2617 (1995).

${ }^{21}$ R. O. Williams, Metall. Trans. 5, 967 (1974).

${ }^{22}$ K. L. Weng, H. R. Chen, Y. R. Yang, Mater. Sci. Eng., A 379, 119 (2004).

${ }^{23}$ T. Ujihara, K. Osamura, Acta Mater. 48, 1629 (2000).

${ }^{24}$ D. Chandra and L. H. Schwartz, Metall. Trans. 2, 511 (1971).

${ }^{25}$ M. K. Miller, Surf. Sci. 246, 434 (1991).

${ }^{26}$ M. K. Miller and M. G. Hetherington, Surf. Sci. 246, 442 (1991).

${ }^{27}$ A. Cerezo, J. H. Hyde, M. K. Miller, S. C. Petts, R. P. Setna, and G. D. Smith, Philos. Trans. R. Soc. London 341, 313 (1992).

${ }^{28}$ J. M. Hyde, A. Cerezo, M. G. Hetherington, M. K. Miller, and G. D. W. Smith, Surf. Sci. 266, 370 (1992).

${ }^{29}$ P. D. Bogdanoff, B. Fultz, and S. Rosenkranz, Phys. Rev. B 60, 3976 (1999).

${ }^{30}$ I. Vincze and I. A. Campbell, J. Phys. F: Met. Phys. 3, 615 (1972).
${ }^{31}$ R. O. Williams, Trans. Metall. Soc. AIME 212, 497 (1958).

${ }^{32}$ L. Reinhard, J. L. Robertson, S. C. Moss, G. E. Ice, P. Zschack, and C. J. Sparks, Phys. Rev. B 45, 2662 (1992).

${ }^{33}$ G. K. Wertheim, V. Jaccarino, J. H. Wernick, and D. N. E. Buchanan, Phys. Rev. Lett. 12, 24 (1964).

${ }^{34}$ B. Fultz, in Mössbauer Spectroscopy Applied to Magnetism and Materials Science, edited by G. J. Long and F. Grandjean (Plenum, New York, 1993), Vol. 1, p. 2.

${ }^{35}$ W. Keune and W. Sturhahn, Hyperfine Interact. 123, 847 (1999).

${ }^{36}$ T. Ruckert, W. Keune, W. Sturhahn, M. Y. Hu, J. P. Sutter, T. S. Toellner, and E. E. Alp, Hyperfine Interact. 126, 363 (2000).

${ }^{37}$ V. J. Minkiewicz, G. Shirane, and R. Nathans, Phys. Rev. 162, 528 (1967).

${ }^{38}$ W. M. Shaw and L. D. Muhlestein, Phys. Rev. B 4, 969 (1971).

${ }^{39}$ W. A. Dench, Trans. Faraday Soc. 59, 1279 (1963).

${ }^{40}$ C. Jiang, C. Wolverton, J. Sofo, L-Q. Chen, and Z-K. Liu, Phys. Rev. B 69, 214202 (2004).

${ }^{41}$ B. Fultz, L. Anthony, J. L. Robertson, R. M. Nicklow, S. Spooner, and M. Mostoller, Phys. Rev. B 52, 3280 (1995). 